\title{
Numerical Study on Noise Reduction of High-Speed Train Based on Non-Smooth Surface
}

\author{
Jiyou HUANG ${ }^{\mathrm{a}}$, Haiyan $\mathrm{ZHU}^{\mathrm{b}, 1}$, Haifei $\mathrm{WEI}^{\mathrm{c}}$, Junhai HUANG ${ }^{\mathrm{a}}$, and Qiying XU \\ ${ }^{a}$ School of Transportation and Logistics Engineering, East China Jiaotong University \\ Nanchang China 330013 \\ ${ }^{b}$ School of Mechanical and Electrical Engineering and Vehicle Engineering, East \\ China Jiaotong University, Jiangxi, Nanchang China 330013 \\ ${ }^{c}$ State Key Laboratory of Traction Power, Southwest Jiaotong University, \\ Chengdu China 610031
}

\begin{abstract}
In this paper, a $350 \mathrm{~km} / \mathrm{h}$ high-speed train is taken as the research object. Using the realizable $\mathrm{k}-\varepsilon$ model to calculate the steady-state flow field around the train, based on the results, calculating aerodynamic noise source of the train body surface by using the broadband noise model. The drag, lift and acoustic data of the train with the non-smooth surface units arranged on different positions of the vehicle are analyzed and compared, so as to analyze the influence of the layout of the non-smooth surface units on the drag reduction and noise reduction of the train. The simulation results show that when the non-smooth surface units are arranged in the bogie area, the aerodynamic resistance of the head and the intermediate vehicle can be effectively reduced, with the drag reduction effect of $12.2 \%$ in the head vehicle and $26.9 \%$ in the intermediate vehicle; when the non-smooth surface units are arranged on the nose of the train, for the intermediate vehicle, the drag reduction effect is $9.3 \%$, and $11.5 \%$ when arranged on the transition area; when the non-smooth surface units are arranged on the nose of the train, there are quite a number of scattered points of low surface acoustic power in the streamline area of the tail vehicle, in which the lowest surface acoustic power level is only $50 \mathrm{~dB}$, which is $25.3 \%$ lower than that of the train without non-smooth surface units.
\end{abstract}

Keywords. High speed train, non-smooth surface, noise reduction, broadband noise

\section{Introduction}

With the continuous improvement of high-speed train speed, the contribution of aerodynamic noise of high-speed train in the total noise is increasing, and it becomes the main source. [1] Therefore, it is necessary to improve the aerodynamic noise of high-speed train by some means.

In nature, there are many organisms with non-smooth surface structure on their body surface. For example, the adaptive skin of shark can be changed into a groove like rib structure to reduce the resistance during swimming. ${ }^{[2]}$ In the aspect of bionic design,

${ }^{1}$ Haiyan Zhu; School of Mechanical and Electrical Engineering and Vehicle Engineering, East China Jiaotong University, Jiangxi, Nanchang China E-mail: zhupetrelcao@163.com 
the bionic non-smooth structure of golf ball surface can make the ball produce whirlpool at high speed, and reduce the pressure difference resistance greatly before and after the ball. [3]

In addition, Liu [4] carried out bionic design on Pantograph members based on the serrated structure of the front wing edge of owl and the groove structure of tidal shellfish, and concluded that the circular corrugated groove can achieve obvious drag reduction and noise reduction effect. Based on the biological characteristics of earthworm body surface, Zhong [5] designed two kinds of non-smooth wall pipes, convex hull type and concave envelope type, which can reduce the friction resistance loss during oil and gas pipeline transportation. Liu [6] studied the micro non-smooth structure of bionic body surface, which can greatly improve the wear resistance of metal cutting tools. In this paper, based on the non-smooth surface element, it is placed on the body surface to change the aerodynamic shape of the high-speed train. Whether the arrangement of the non-smooth surface element can improve the aerodynamic noise of the train and the influence on the aerodynamic resistance and aerodynamic lift of the train are studied.

\section{Calculation Model and Method}

\subsection{Calculation Method}

Since the aerodynamic noise of high-speed train is continuously distributed in a wide frequency range, the k- $\varepsilon$ model can be used to calculate the turbulence parameters, and broadband noise models can be used to integrate the surface unit or the volume unit to obtain the noise power level.

Using the Broadband Noise Sources of fluent noise model, to obtain the noise sources information of train surface. Based on the steady-state flow field, using acoustic power and surface acoustic power to reflect the aerodynamic noise around the train. The acoustic power represents the noise power per unit volume generated by isotropic turbulence of train flow field. The surface acoustic power represents the aerodynamic noise power generated by boundary layer turbulence on the train surface per unit area. This paper calculates the body noise sources of the train with non-smooth surface units at the nose, bogie area, and the transition area between streamline and non-streamline of the head vehicle and the tail vehicle at the speed of $350 \mathrm{~km} / \mathrm{h}$.

\subsection{Calculation Model and Boundary Condition}

The basic element of the non-smooth surface used in this paper is a convex spherical element with a center angle of $120^{\circ}$ and a radius of $3 \mathrm{~mm}$, which is distributed on the top of a square with a side length of $12 \mathrm{~mm}$. Since the noise sources are mainly distributed in some areas where the curvature of the vehicle body changes greatly [710], this paper establishes the model according to a certain reduction ratio of a certain type of vehicle, and makes appropriate simplification. Several non-smooth surface units are arranged at the nose of the train, the bogie area and the transition area between streamline and non-streamline. The arrangement of them are shown in Figure 1. In order to simplify the calculation, the calculation domain is half of the actual situation. The total length of the half vehicle model is $7.5 \mathrm{~m}$, in which the length of each is $2.5 \mathrm{~m}$, the vehicle height is $0.355 \mathrm{~m}$ and the half vehicle width is $0.159 \mathrm{~m}$; the half basin is $45 \mathrm{~m}$ 
$\times 24 \mathrm{~m} \times 36 \mathrm{~m}$, and the symmetry plane of the train coincides with the symmetry plane of the drainage basin, and the distance between the nose of the train and the entrance of the flow domain is $7.45 \mathrm{~m}$. The type of mesh is hybrid mesh, unstructured mesh is near the surface of the body, and the rest is structural mesh. After the mesh calculation is completed, fluent is imported to calculate the steady flow field around the $350 \mathrm{~km} / \mathrm{h}$ high-speed train with non-smooth surface units at different positions based on $\mathrm{k}-\varepsilon$ model. The mesh quantity is shown in Table 1.
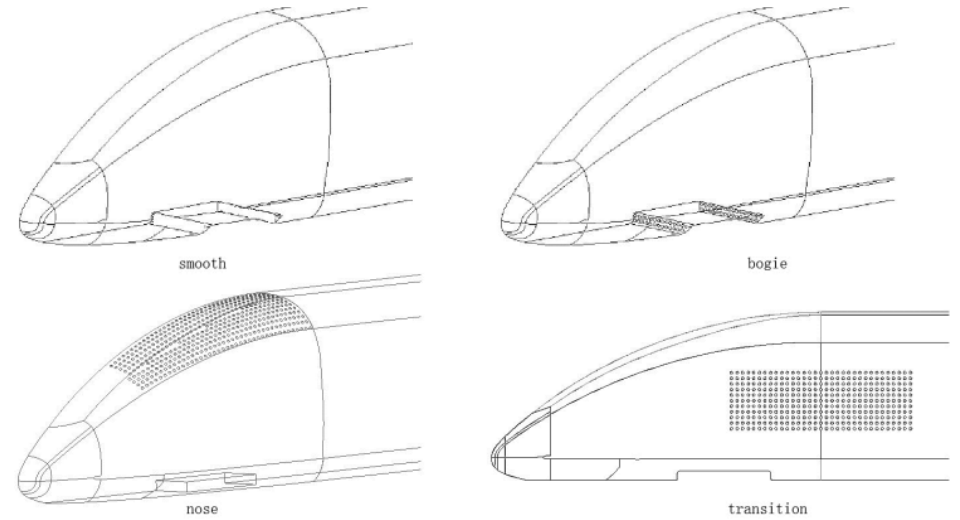

Figure 1. Position of non-smooth surface units.

Table 1. Mesh quantity.

\begin{tabular}{ccccc}
\hline position & smooth & nose & bogie & transition \\
\hline quantity & 8759026 & 13668206 & 11631567 & 6866750 \\
\hline
\end{tabular}

\section{Calculation Results}

\subsection{Drag and Lift Characteristics}

The drag and lift simulation data of the head, the tail and the intermediate vehicle are calculated respectively when the non-smooth surface units are arranged at different positions, and the data are made into a histogram, as shown in Figure 2.

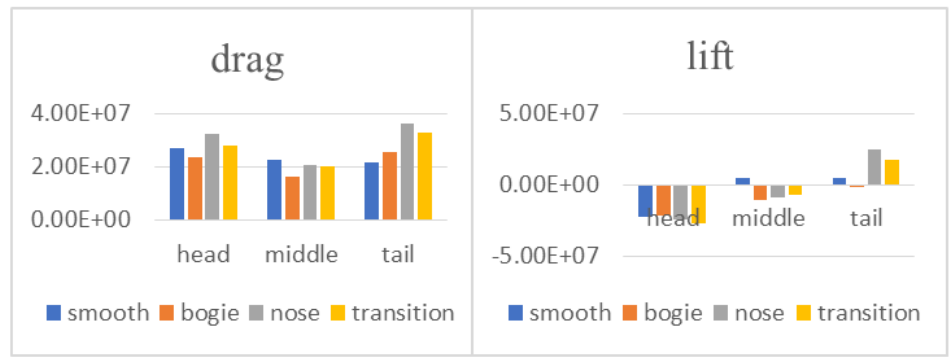

Figure 2. The drag and the lift of vehicles.

It can be seen from Figure 2 that the arrangement of non-smooth surface units has a great impact on changing the air resistance of vehicles, and the arrangement of it that arrange at different positions can increase or decrease the resistance. This shows that 
the aerodynamic shape of the train has a great influence on the change of aerodynamic resistance of vehicles, which is in line with the current academic research.

According to the information in the figure, when the non-smooth surface units is arranged in the bogie area, the drag reduction effect of the head vehicle is $12.2 \%$, and that of the intermediate vehicle is $26.9 \%$; there are some reduction effect in intermediate vehicle when the non-smooth surface units are arranged in the nose and transition area, the former is $9.3 \%$ and the latter is $11.5 \%$, in other cases, the aerodynamic drag not only doesn't decrease but also increases. This shows that when they are arranged correctly, non-smooth surface units have obvious effect on reducing the aerodynamic resistance of the vehicle. The calculation formula of drag reduction effect is

$$
\text { force reduction effect }=\frac{F-F^{\prime}}{F} * 100 \%
$$

\section{$\mathrm{F}=$ force of smooth surface vehicle \\ $\mathrm{F}^{\prime}=$ force of non - smooth surface vehicle}

As for the lift of vehicle, it can be seen from Figure 2, for the head vehicle, the lift is negative, and the change by non-smooth surface units of lift is not obvious; for intermediate vehicle, the lift is positive generally, and negative when arranged with non-smooth surface units; for tail vehicle, the lift is positive and that of train with nonsmooth surface units in bogie area is negative, in the other two cases, the value of lift is positive and large, which may be detrimental to the stability and stability of train operation.

\subsection{Acoustic Power Distribution}

After the steady-state flow field calculation is completed, the Broadband Noise Sources model is opened to calculate the noise source, in order to obtain the acoustic power level distribution scatter diagram of the outer contour line of the longitudinal symmetry plane of the train. The horizontal axis of the scatter diagram is the train length direction, and the longitudinal axis is the acoustic power level as shown in Figure 3.

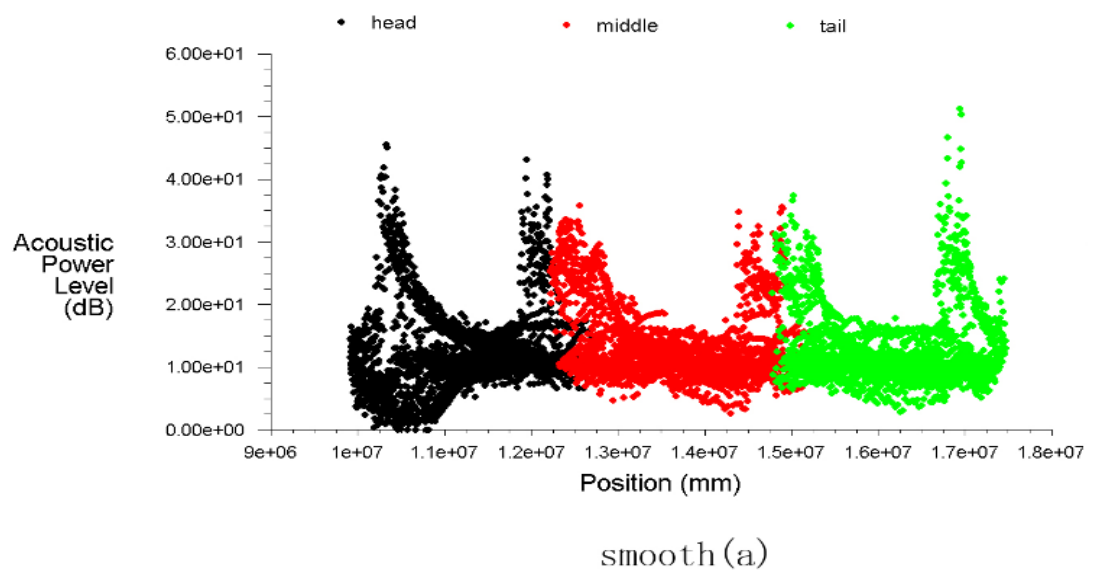




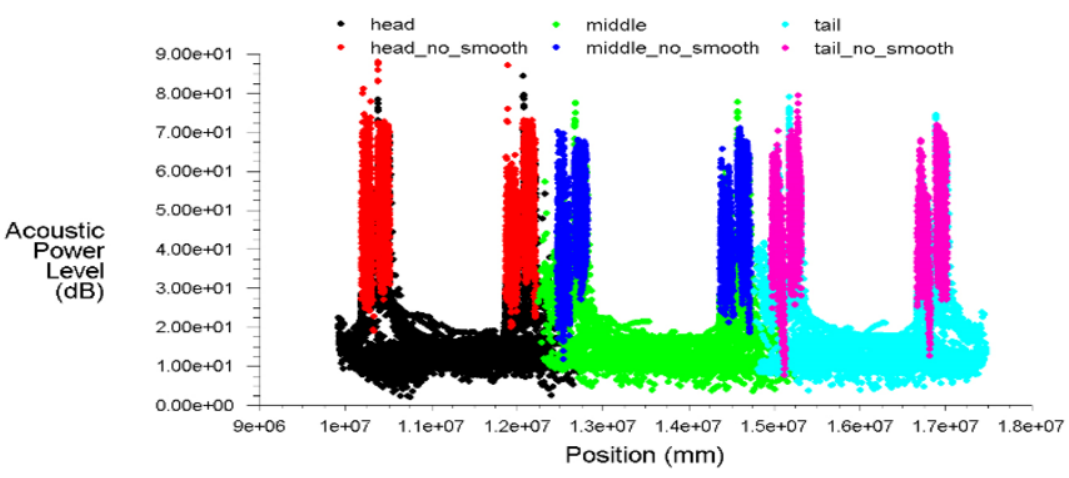

bogie (b)

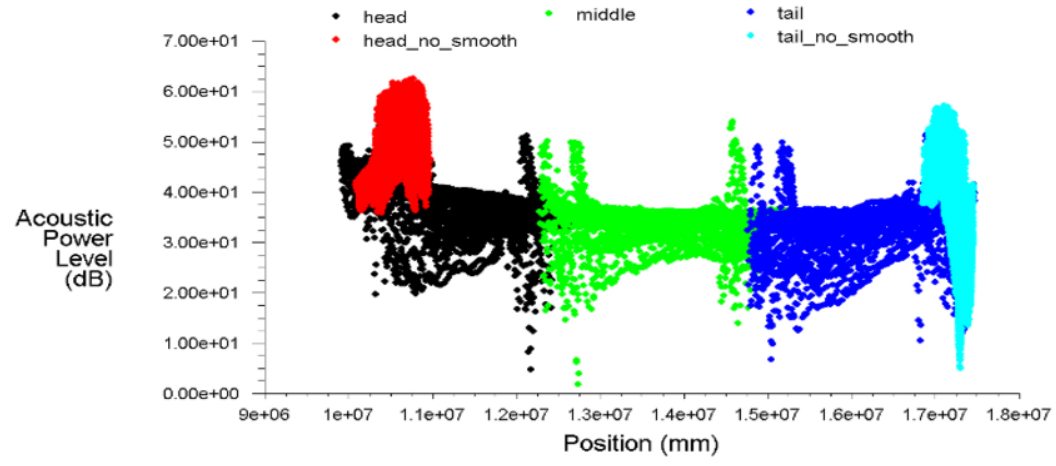

nose (c)

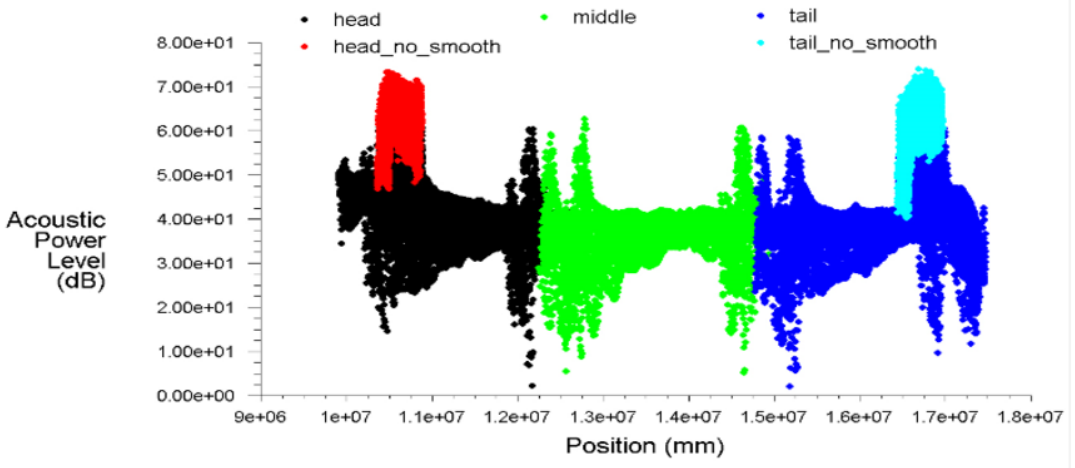

transition $(d)$

Figure 3. Acoustic power distribution

After analyzing comprehensively, the result of the acoustic power level of the train that are arranged with non-smooth surface units and nothing show that non-smooth surface units could increase the acoustic power level, when the non-smooth surface units are arranged in the bogie area, the level in the bogie area increases significantly, 
while is consistent with that of the smooth body in other areas roughly. Therefore, it can be inferred that the arrangement of non-smooth surface units on the body surface has a great influence on the turbulence in the flow field space and is unfavorable to reduce the noise. Therefore, it has no effect on the aerodynamic noise produced by isotropic turbulence. The maximum acoustic power level data are shown in the Table 2.

Table 2. Maximum acoustic power level

\begin{tabular}{|c|c|c|c|c|}
\hline position & smooth & nose & bogie & transition \\
\hline Acoustic power (dB) & 51.3 & 69.4 & 88.2 & 78 \\
\hline
\end{tabular}

\subsection{Surface Acoustic Power Distribution}

Compared with Figure 4-a and Figure 4-c, it can be seen that when the non-smooth surface units are arranged at the nose, the surface acoustic power of the nose of the head vehicle is increased significantly, but there are lower surface acoustic power level scatter points at the same position than that of the smooth surface. Compared with the nose of the tail vehicle, there are quite a number of scattered points with low value. The lowest value is only $50 \mathrm{~dB}$, which is $25.3 \%$ lower than that of the smooth nose's which is $67 \mathrm{~dB}$, this shows that the arrangement of non-smooth surface units at nose of the vehicle is effective to decease the surface acoustic power level. In other cases, the surface acoustic power level not only doesn't decrease but increases.
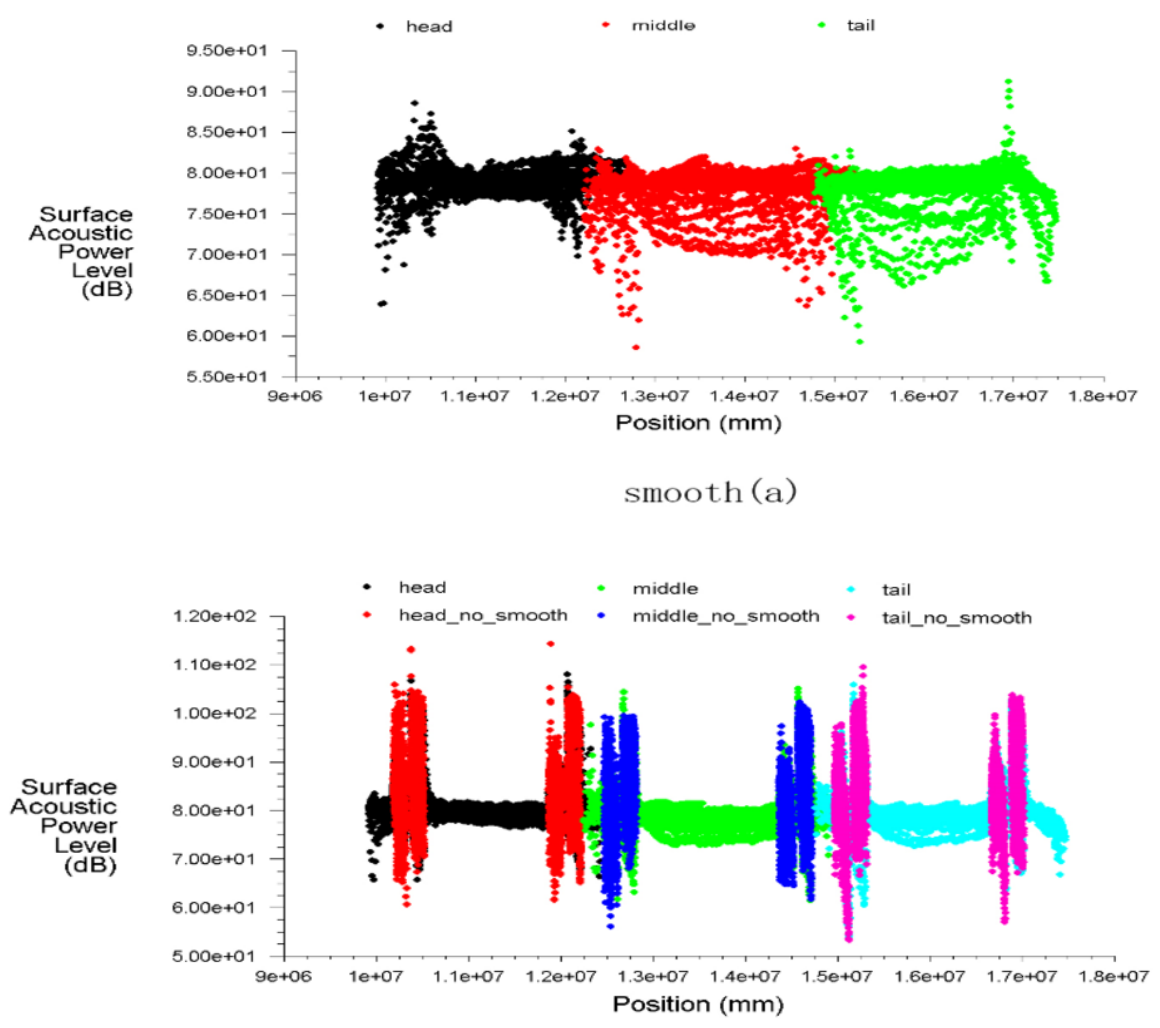

bogie (b) 


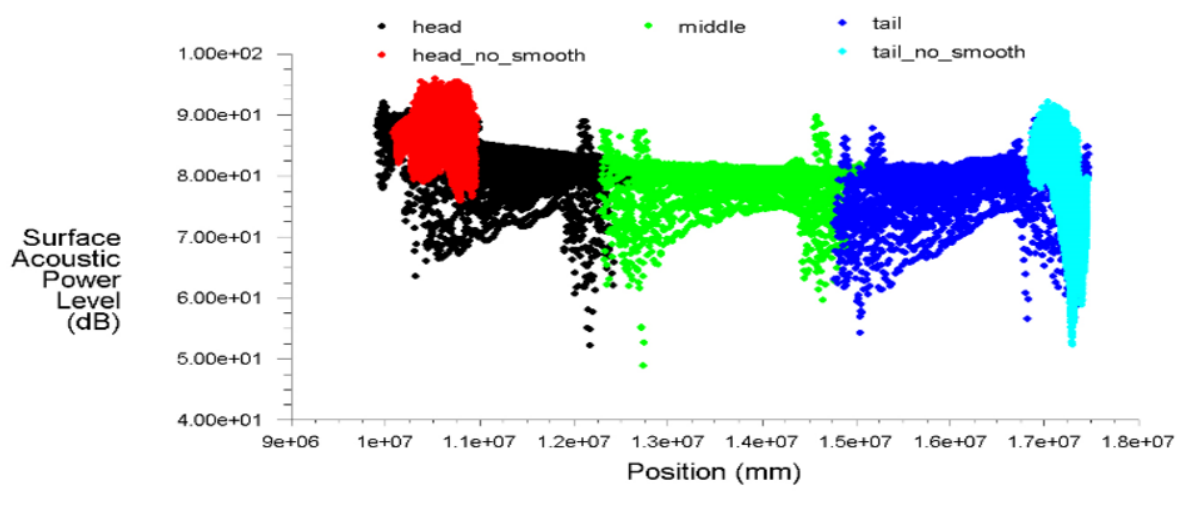

nose (c)

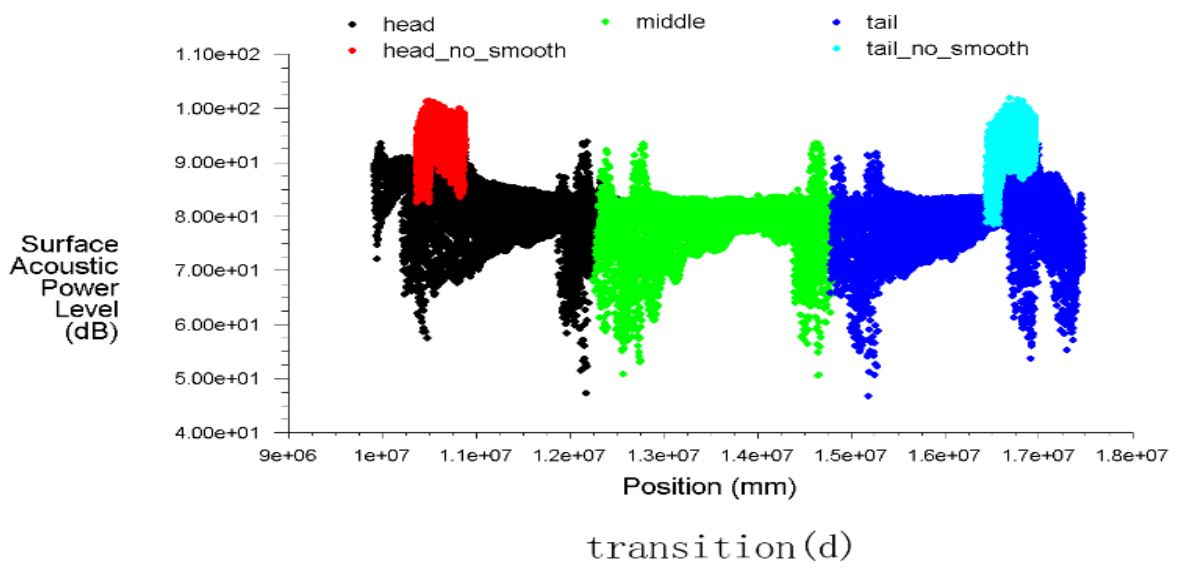

Figure 4. Surface acoustic power distribution

Therefore, it is an effective method to reduce the surface acoustic power generated by boundary layer turbulence by arranging non-smooth surface units at appropriate positions on the train.

\section{Conclusion}

In this paper, based on the non-smooth surface units, which are arranged in different positions of the vehicle surface. Under the speed of $350 \mathrm{~km} / \mathrm{h}$, the drag, lift and broadband noise of the train with non-smooth surface units are analyzed. The conclusions are as follows.

- The aerodynamic drag can be effectively reduced in the head and intermediate vehicle when non-smooth surface units are arranged in the bogie area, the drag reduction effect of the former is $12.2 \%$, and that of the latter is $26.9 \%$

- The aerodynamic drag can be effectively reduced in intermediate vehicle when non-smooth surface units are arranged in the nose and transition area, the drag reduction effect of the former is $9.3 \%$ and the latter is $11.5 \%$ 
- When the non-smooth surface units are arranged in the nose and transition area, the lift of the tail vehicle will be too big to be conducive to driving safety.

- When the non-smooth surface units are arranged in the nose of the vehicle, there are quite a number of scattered points with low surface acoustic power level at the nose of the tail vehicle, the minimum level is only $50 \mathrm{~dB}$, which is $25.3 \%$ lower than that of the smooth nose's which is $67 \mathrm{~dB}$.

Therefore, under the condition of ensuring safety of high-speed train, when the location of non-smooth surface unit is arranged appropriately, the non-smooth surface unit can play a role in reducing drag and noise.

\section{Acknowledgements}

The authors would like to thank anonymous reviewers for their helpful comments and suggestions to improve the manuscript. This research was supported by Jiangxi Provincial Natural Science Foundation (Grant No. 20202ACBL204008 and 20181BAB206025), Science and technology project of Jiangxi Education Department (Grant No. GJJ190330, GJJ190333 and GJJ190308).

\section{References}

[1] Liu JL, Zhang JY, Zhang WH. Numerical analysis on the aerodynamic noise of the high-speed train head. Journal of the China Railway Society.2011 Sept;33(9):19-25.

[2] Wu MK. Application and development of the imitation shark skin swimsuits. Progress in Textile Science \& Technology.2009(2):90-91

[3] Wu J. Starting from the pockmarked golf ball -- sniping force and lifting force of moving objects in fluid. Mechanics in Engineering.2005.5(27):88-92

[4] Liu HT, XZ. Study on drag and noise reduction of pantograph rods based on bionic non-smooth structures. Noise and Vibration Control.2018 Apr;Z1(38):269-272.

[5] Zhong PS, Zhao XH, Ni W, Yang KG. Design and finite element analysis of bionic mon-smooth surface pipes. Chinese Hydraulics \& Pneumatics.2019(07):70-75.

[6] Li XQ. Study on drag reduction characteristics and flow field structure of bionic non-smooth surface mimicked puffer spines. Master, Jiangsu University of Science and Technology, Zhenjiang, Jiang Su, 2019 Jun.

[7] Kitagawa T, Nagakurak K. Aerodynamic noise generated by Shinkansen cars. Journal of Sound andvibration, 2000, 231(3): 913-924.

[8] Nagakura K. Localization of aerodynamic noise sources of Shinkansen trains. Journal of Sound and Vibration, 2006, 293(3 -5): 547-556.

[9] Ito M. Improvement to the aerodynamic characteristics of Shinkanen rolling stock. Proceedings of the Institution of Mechanical Engineers, Part F: Journal of Rail and Rapid Transit, 2002, 214(3):135-141.

[10] Zhang J, Sun BC, Guo T, Fang J, Zhao WZ. Research on aerodynamic noise radiated from whole body surface of high-speed train and its distribution. Journal of the China Railway Society.2015 Feb;37(2):10-16. 